\title{
Educational Software Interface for Power Electronic Applications
}

\author{
J. A. Sanchez, J. I. Leon, L. G. Franquelo, J. M. Carrasco, S. Vazquez, R. Portillo and E. Dominguez \\ Electronic Engineering Department, School of Engineering \\ University of Seville \\ Seville, Spain 41092 \\ Email: shanshe@gte.esi.us.es
}

\begin{abstract}
The design of friendly user tools for power electronics applications is a hot topic among the researchers all over the world. In fact, the digital signal processors programming is a hard task which requires a deep knowledge of the specific hardware platform where the user control program is implemented. In this paper, a user interface for programming digital signal processors (DSP) to control a power electronic converter is presented. The proposed program, called jscomm, is currently used as a useful tool to understand how the DSP is programmed, to control a power converter safely and to monitor all the interesting variables of the power converter operation.
\end{abstract}

\section{INTRODUCTION}

The simulation and experimental testing of power systems is a fundamental topic of electrical and electronic engineers all over the world [1]-[8]. In this way, different simulation softwares for understanding power electronics have been developed in order to facilitate the teaching in such a difficult topic for new or non-expert students and researchers. As one of the most interesting current simulation tools, the interactive power electronics seminar (IPES) software is a simple educational module dedicated to basic power electronics circuits [9]. Using this tool or other similar, the students can learn the basic operation of many power converter topologies, signal theory in power electronics, pulse width modulation and space vector modulation, etcetera.

On the other hand, recently the e-learning concept has been also successfully applied to the power electronics teaching achieving good results [10], [11]. Using e-learning platforms, the student/researcher can operate a hardware system usually via web. In this way, the user is working with a distant real prototype and is operating it online. The e-learning concept is very useful where a high number of users have to actuate with a single hardware prototype. However, only by simulations or using the e-learning, the students and/or researchers do not actually work with real prototypes and they do not notice the hardware/software problems related with real applications of power converters. They do not see with their own eyes the converter operating, hear the switching noise or work with real power active and passive devices.

In this way, the use of laboratory prototypes especially designed and built for educational purposes is very useful. Using this kind of prototypes, the users are closer to the real power system design dealing with problems such as the power devices sizing, the measuring errors effects, the switching noise, etcetera. Several user tools are required in order to facilitate the interface between novel users and a complex power system formed by the power converter experimental setup, the measuring system and the control hardware (usually a digital signal processor (DSP)). These user tools can be developed by an unique software which implements an interface carrying out all the necessary operations under safe conditions.

\section{THE PROPOSED USER INTERFACE $(j$ scomm $)$}

In this paper, a friendly user interface for DSP programming and control of power electronic converters is presented. The software, called jscomm has been developed in the Power Electronics Research Group at the University of Seville. The jscomm software is currently being used for post-graduate students in order to develop, test and adjust control techniques for power electronic converters as a part of the required knowledge to get the $\mathrm{PhD}$ degree. It is being applied to medium-high power systems but it can be also used for low power applications.

The jscomm software objectives are:

- The jscomm can operate independently of the specific DSP platform by Texas Instruments. In this way, the user can carry out the tests avoiding a deep knowledge of the hardware.

- Using the jscomm, the necessary state machine to control a power converter can be easily developed and monitored during its operation.

- The controller of the power converter can be tuned and tested observing the influence of the controller constants on the power converter operation.

- Using the oscilloscope tool present in the jscomm, it is also possible to monitor any control or measured variable. This facilitates the user $\mathrm{C}$ code debugging finding possible errors in the implemented control techniques.

\section{A. The oscilloscope tool}

The jscomm provides an oscilloscope plotting any variable of the power system (the number of channels is configurable). In this way, the operation of the power converter can be monitored online and the implemented control technique can be tested safely. A snapshot of the oscilloscope present in the jscomm is represented in Fig. 1. It is important to notice that not only the physical variables of the prototype can be 


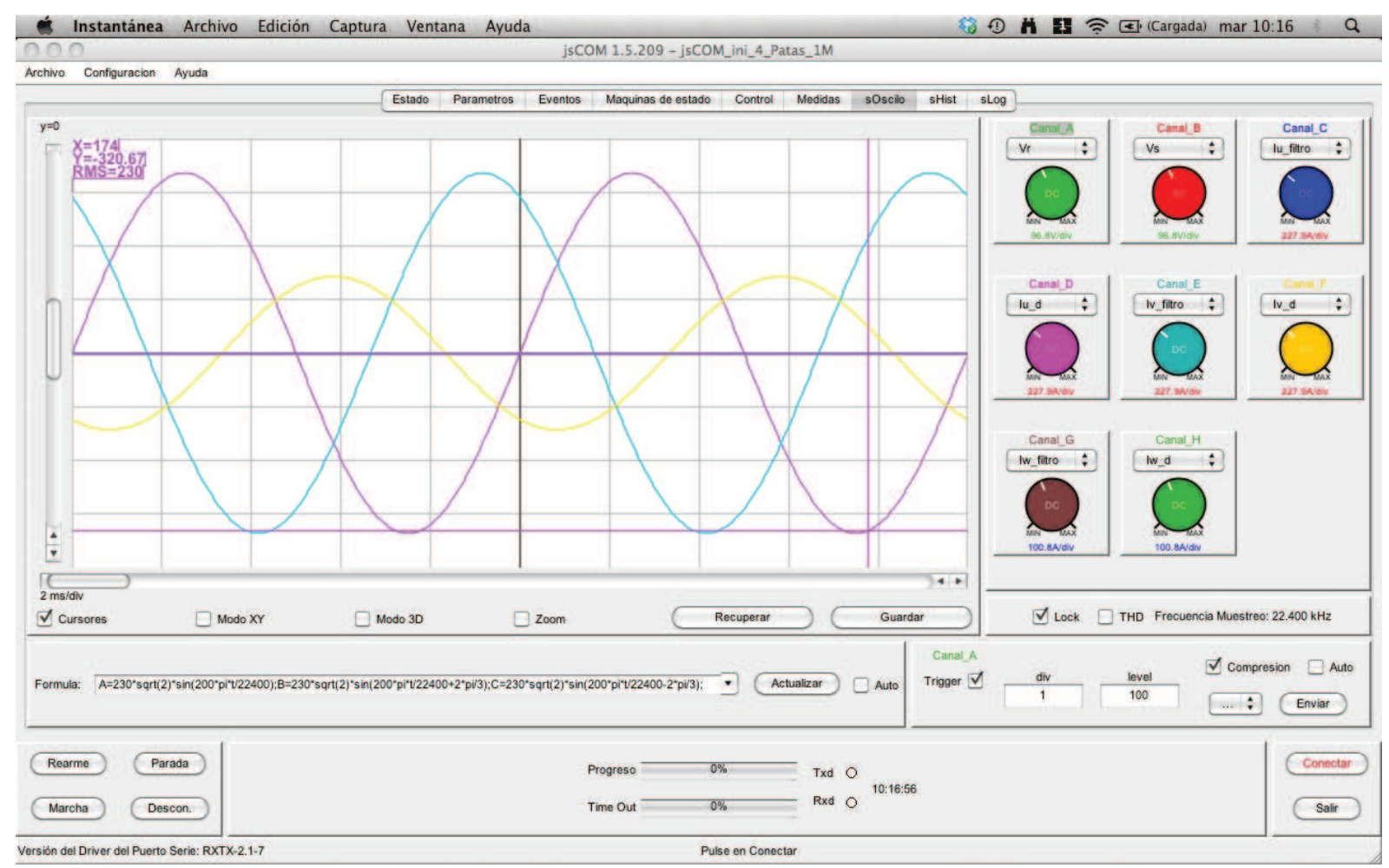

Fig. 1. Eight channels oscilloscope where control or measured variables can be plotted online.

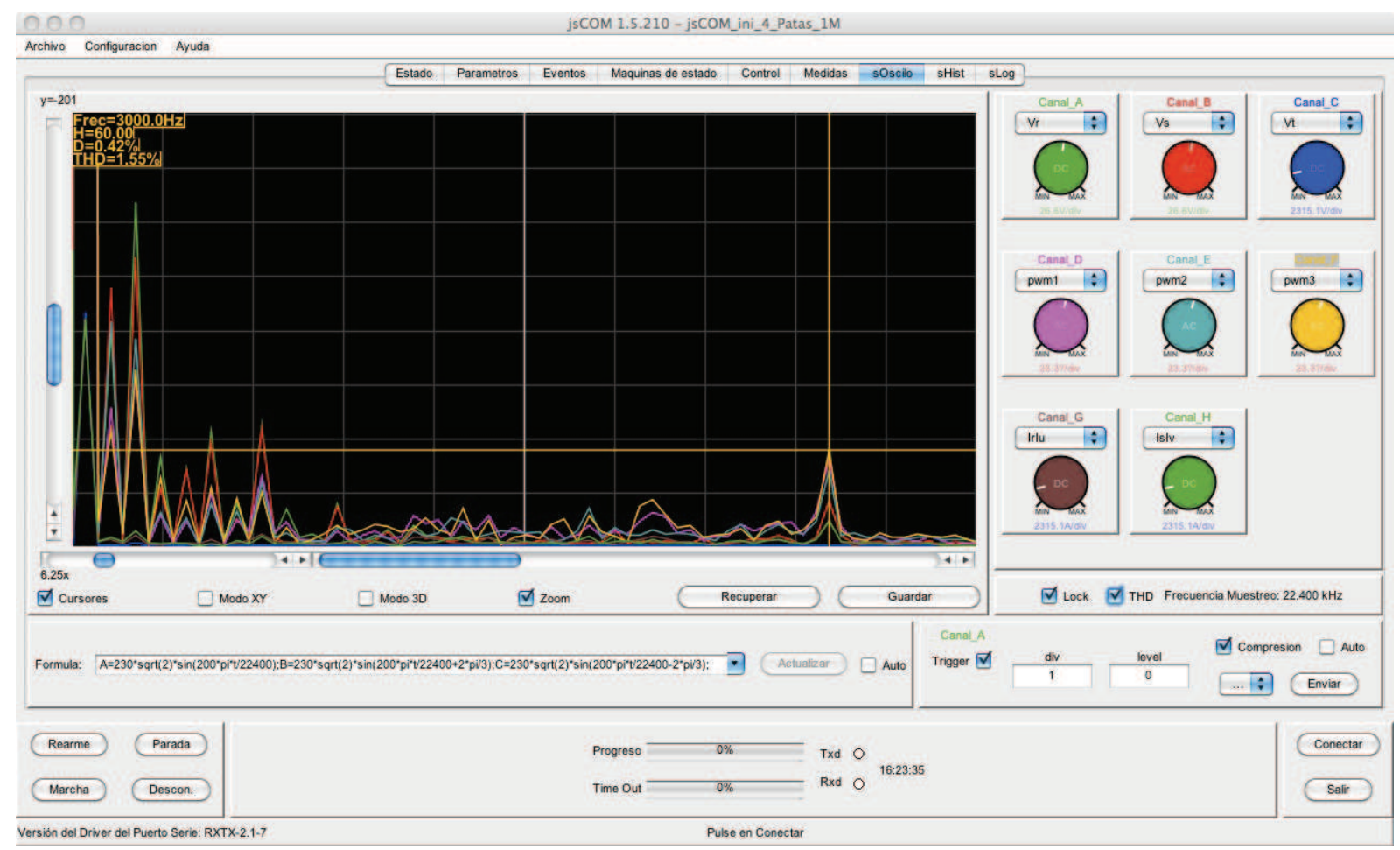

Fig. 2. Oscilloscope tool representing the harmonic spectrum of a measured variable.

represented but also the auxiliary programming parameters. This capability for tracing any DSP control program internal variable facilitates the debugging procedure and the under- standing of the power converter behavior. The oscilloscope tool provides important information such as the RMS value and the average value of each channel. As usual oscilloscopes, 


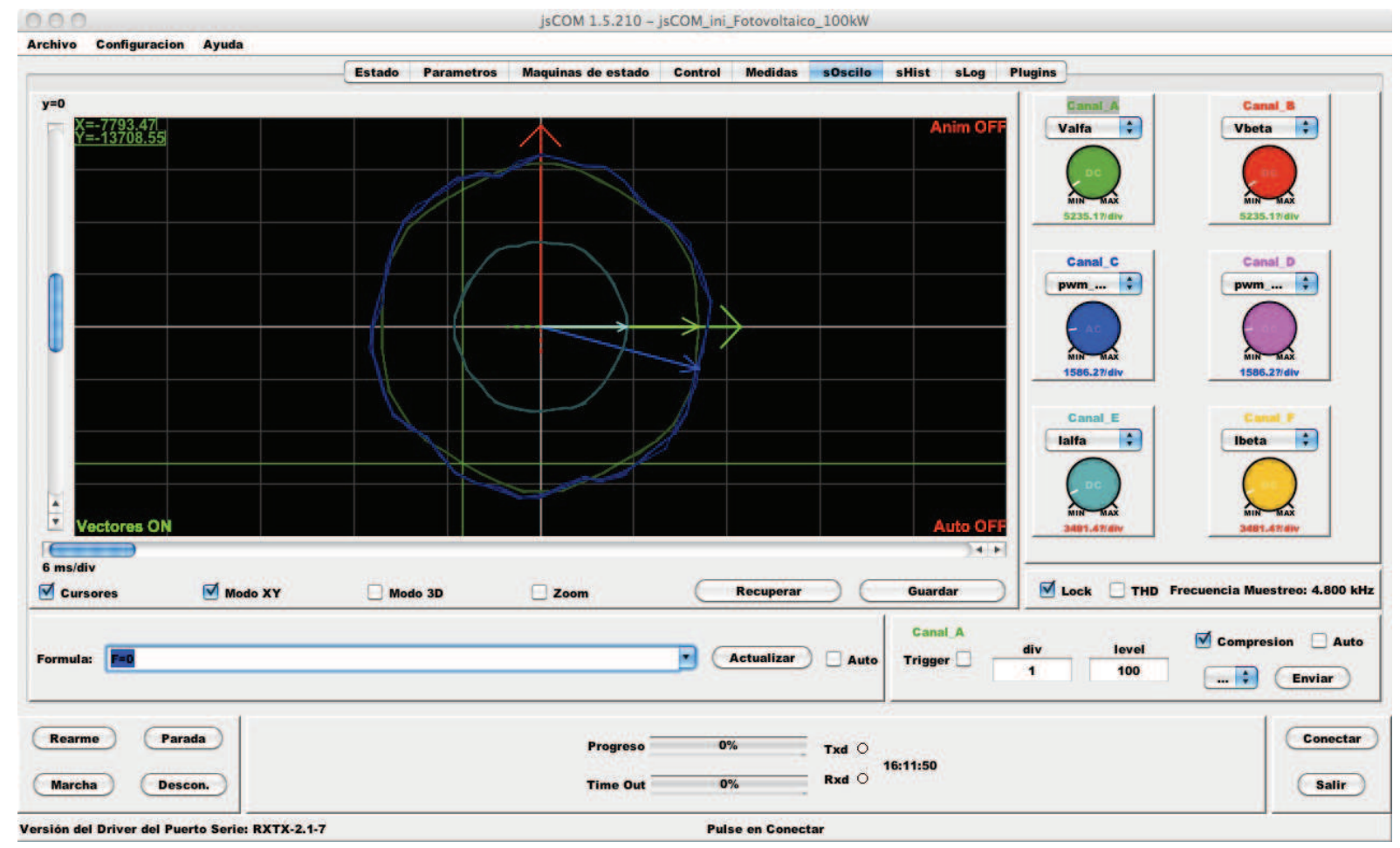

Fig. 3. Oscilloscope tool configured in the XY representation mode.

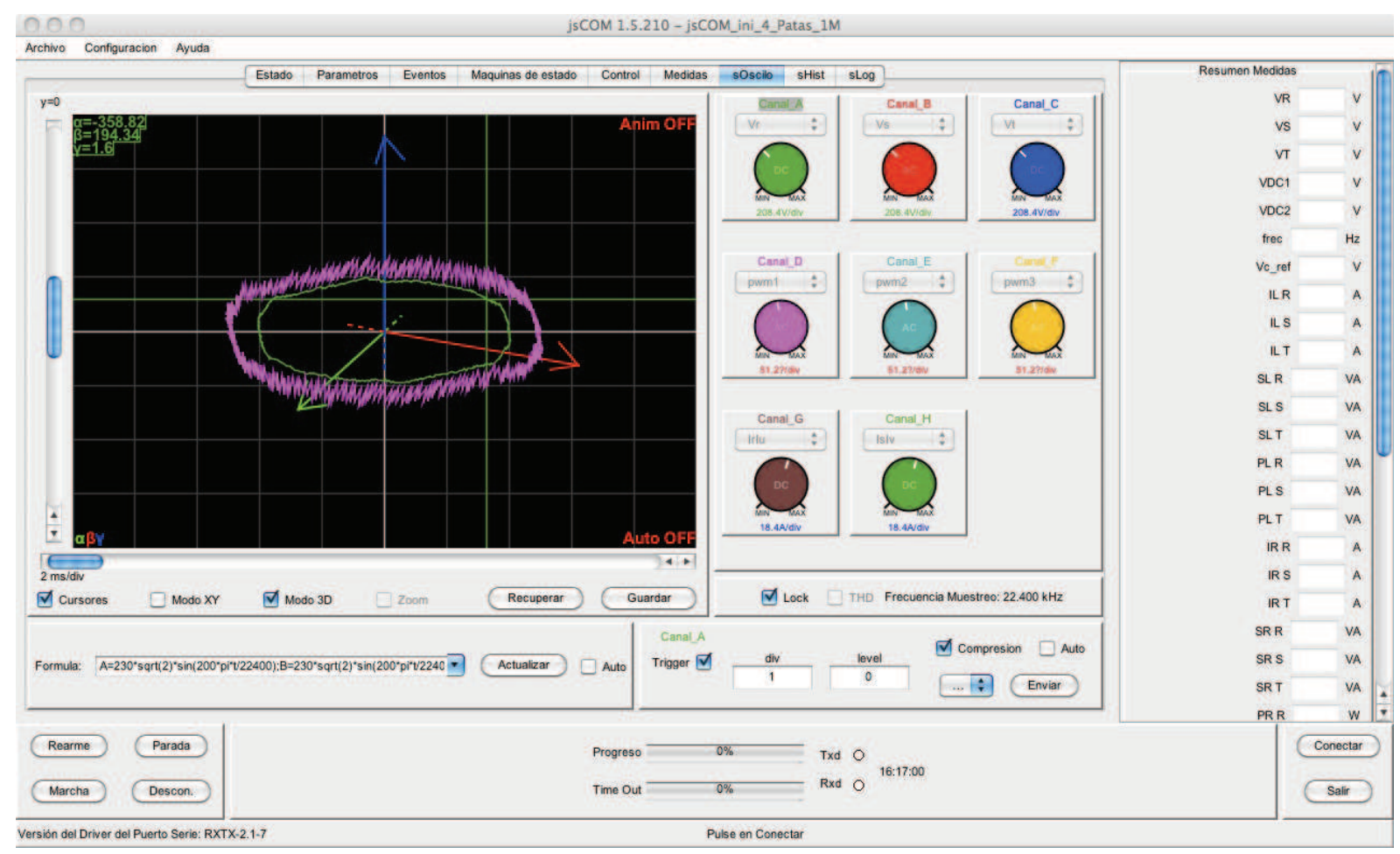

Fig. 4. Oscilloscope tool configured in the three-dimensional representation mode.

it provides the trigger function which is useful to capture the waveforms when a condition is fulfilled. In addition, the volts/div, amp/div and time/div in the jscomm oscilloscope have exactly the same functionality compared with real os- cilloscopes. As an additional function, it is possible to carry out mathematical operations using the oscilloscope channels as a sum, subtraction, multiplications, etcetera. In this way, it is possible to calculate operations such as $A=A+B-C+3 * D$ for 
instance (where A, B, C and D are the four first oscilloscope channels). Besides, as an additional functionality, the harmonic spectrum can be also plotted providing important information for power systems and this is shown in Fig. 2.

Finally, two representation options can be defined in the jscomm oscilloscope tool. Firstly, the XY representation mode can be defined representing channel A versus channel B. The XY representation mode is useful in power electronics system to represent variables in alfa-beta coordinates. A representation of the jscomm oscilloscope working in XY mode is shown in Fig. 3 where a phase current of a power converter is represented in the alfa-beta plane representing the beta current versus the alfa current. Secondly, a threedimensional representation mode can be also chosen. In this way, three different channels can be represented and it is useful to represent for instance the phase currents of the power converter in in a cartesian coordinates frame. The threedimensional representation mode is shown in Fig. 4 where the phase currents of a three-phase power converter are plotted. These two representation modes help to the user to understand how the power converter is operating and the influence of the controller on the power converter operation. Finally, it has to be noticed that these representations are plotting information continuously using a trail to represent past samplings as can be observed in Fig. 3 and Fig. 4.

\section{B. The states diagram tool}

One of the most interesting characteristics of the jscomm is that it includes a tool for developing a states machine to control the power system. The states machine is plotted using a classical diagram where the states are represented using balls and the conditions to carry out the transitions between the states are defined using arrows. As a Moore machine is implemented, each state has defined outputs and the transitions are combinations of the inputs or other conditions written on each arrow. The transitions can be defined as:

- User commands such as stop or connect related to buttons present in the jscomm user screen.

- Measurements such as the dc voltage, excessive phase currents, emergencies, etcetera.

- A time condition defining therefore transitory states.

- A combination of the previous ones.

An example of the generation of the states machine for a conventional three-phase power converter rectifier is represented in Fig. 5.

It is very important to notice that the states machine diagram, developed by the user with a very friendly software interface, directly generates the corresponding $\mathrm{C}$ code to be implemented in the DSP. In this way, the generation of the states machine, needed in most of the power system applications, is simplified. In fact, the use of the states machine diagram avoids design errors and, during the converter operation, the state where the converter is working each moment is highlighted.

\section{The calibration tool}

An important topic of the power conversion is the calibration of the voltage and current sensors. This issue has to be carried out minimizing the possible measuring errors and the

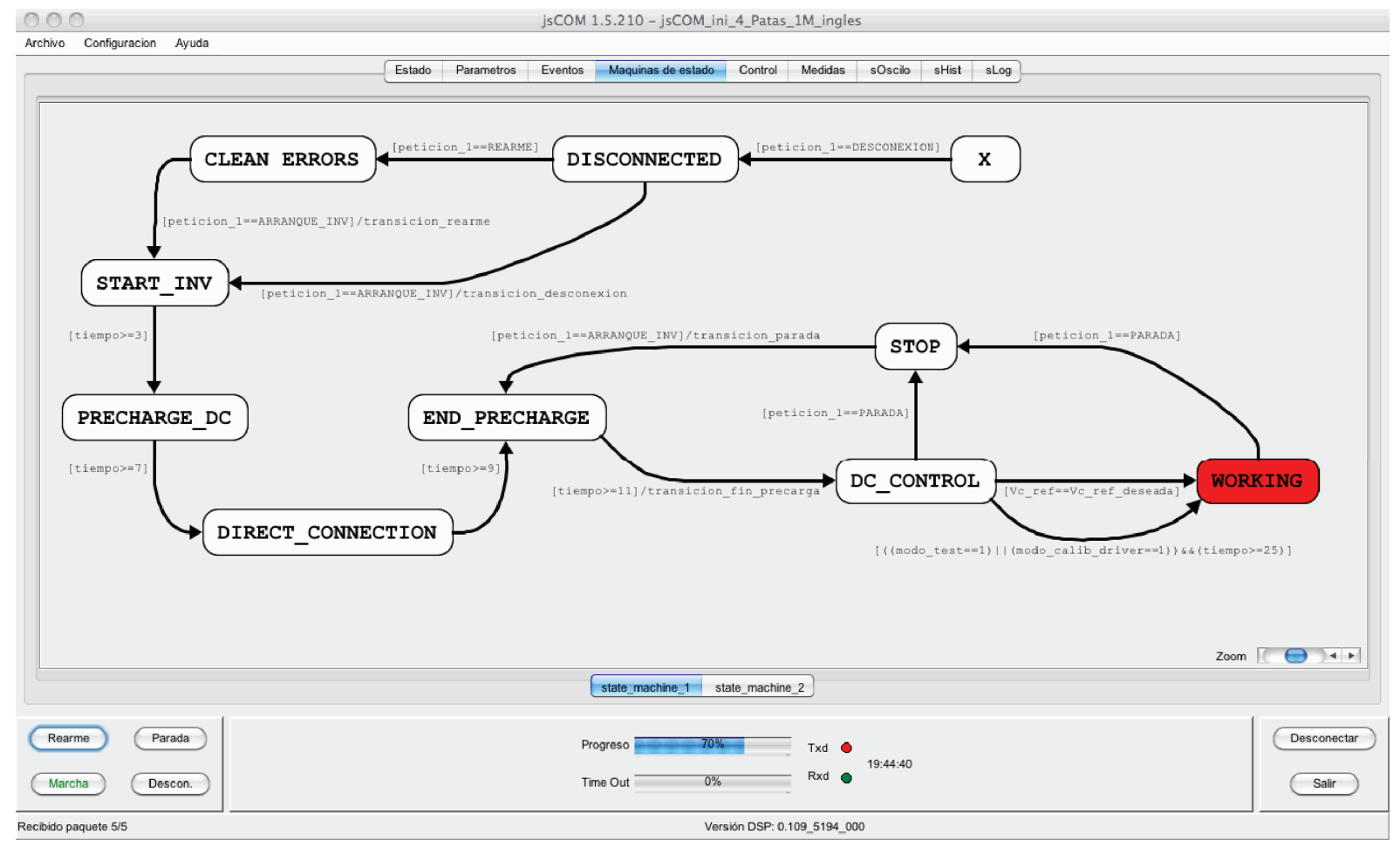

Fig. 5. States machine screen where the possible states and the transitions are defined. 


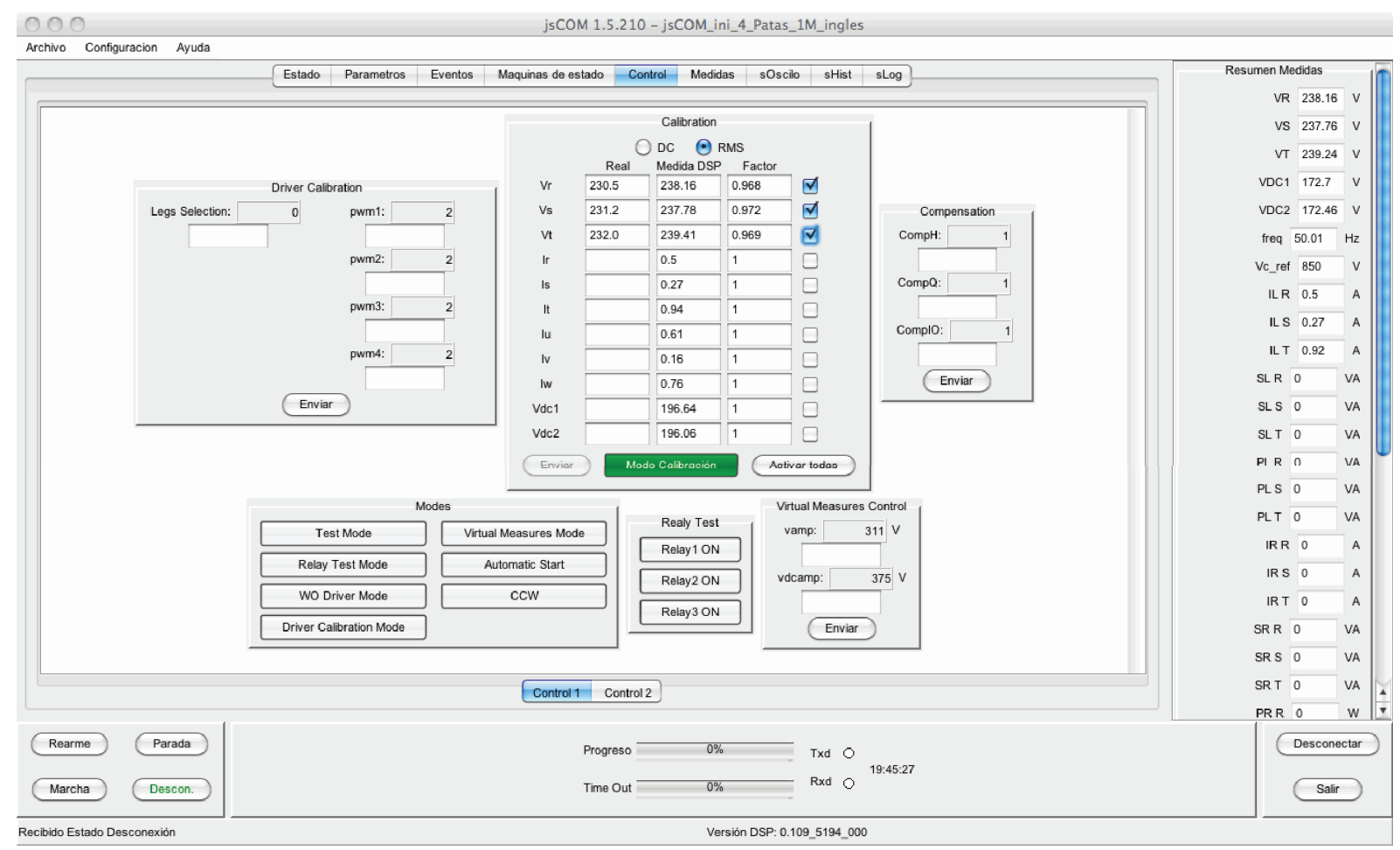

Fig. 6. Calibration tool where the measurement adaptation parameters are determined to reduce the calibration errors.

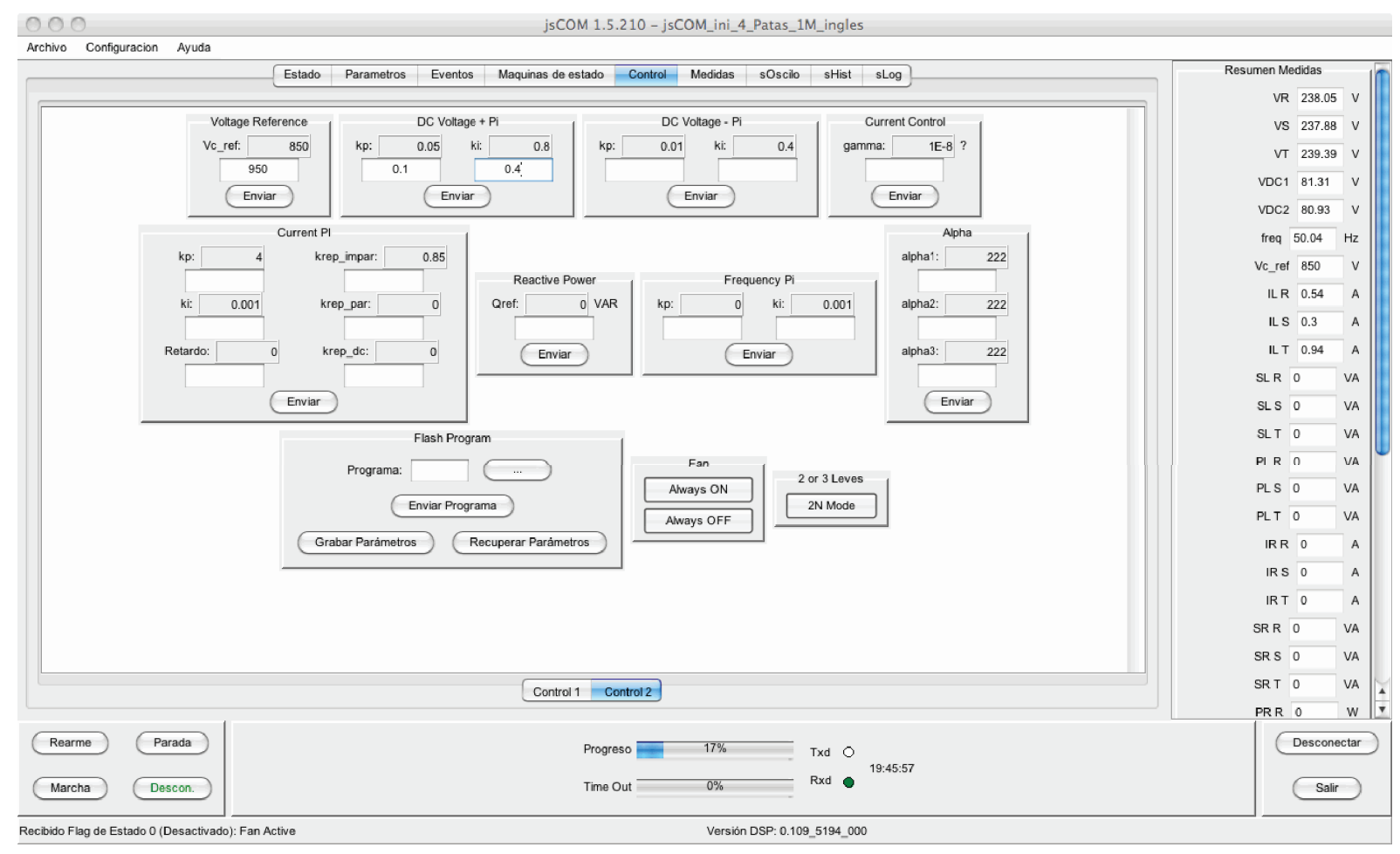

Fig. 7. Controller screen where the tuning of the controller constants can be done.

jscomm software provides an automatic calibration tool to solve it. All the measurements of the power system can be calibrated online. The user has to introduce in the jscomm calibration screen the actual value of the measurement and execute the automatic self-calibration tool. The self-calibration procedure calculates the measurement adaptation parameters to reduce the measurement errors. Finally, once the power converter sensors have been calibrated, the measurement adap- 
tation parameters can be saved in a file for next experiments. In this way, the calibration procedure has to be executed only the first time and it is not necessary to repeat all the process. The jscomm calibration tool is represented in Fig. 6 .

\section{The controller adjustment tool}

The design of high performance controllers for power converters is a hard task for non expert students/researchers. A lot of efforts are usually spend on the adjustment of the controller parameters and the determination of the dynamic and steadystate behavior of the power system. Typically the optimal controller parameters do not coincide with those optimal for the real prototype due to small differences such as voltage drops, losses, passive device tolerances, noise, calibration errors, etcetera. In this way, it is always necessary a tuning procedure to obtain the best possible performance of the power converter.

The jscomm users can design any control technique to be implemented in the power converter. The $j$ scomm permits to change the values of the controller constants during the operation of the converter making easier the controller constants tuning process. This fact can show the impact of any term of the controller on the performance of the control variables. For instance, considering a classical controller where an external voltage control loop and an internal current control loop are used, the influence of the proportional, integral and derivative PID controllers on the dc voltage or the phase currents can be measured. This can help to novel power control researchers to understand the operation of the power converter and the controllers design. A snapshot of the controller adjustment tool present in the jscomm software is presented in Fig. 7.

\section{E. The emergency management tool}

An special attention has to be paid on the emergencies management. The main concern in a educational power system is the safety and all the possible emergencies have to be taken into account. This emergency could be an overvoltage, overcurrent, minimum voltages in the dc side, driver errors, communication errors, etcetera. If an emergency happens, the jscomm software stops the power system operation and shows the emergency cause to the user highlighting the corresponding flag in the emergency tool. This tool is shown in Fig. 8.

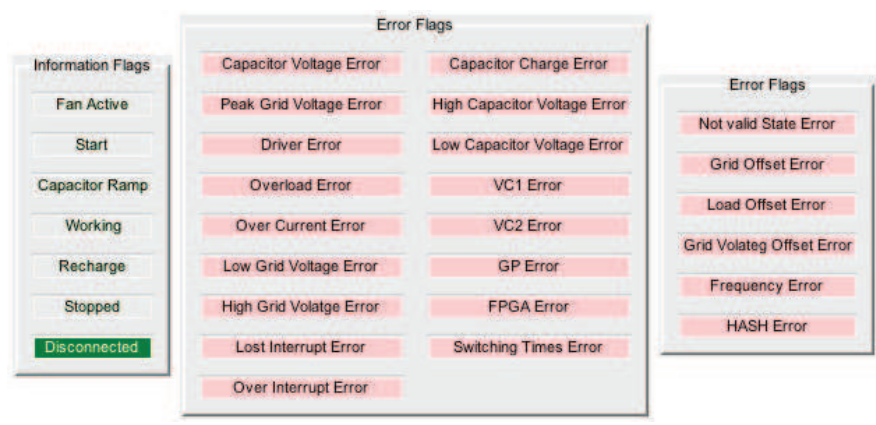

Fig. 8. Emergency tool showing the possible emergencies in the power system

\section{F. Auxiliary jscomm tools}

Some extra functionalities have been added to the jscomm program. These auxiliary tools are:

- FFT calculation.

- Data compression in the communication between the DSP and the jscomm.

- Log file generation including a historical information about all the operations carried out in a jscomm session.

- Flash memory programming.

\section{CONCLUSIONS}

In this paper, a user interface, called jscomm, for programming digital signal processors (DSP) to control a power electronic converter is presented. The jscomm software is currently being used for post-graduate students in order to develop, test and adjust control techniques for power electronic converters as a part of the required knowledge to get the $\mathrm{PhD}$ degree. All the tools provided by the $j$ scomm program have been introduced during the manuscript.

\section{ACKNOWLEDGMENT}

The authors gratefully acknowledge financial support provided by the Spanish Ministry under Project DPI2001-3089, DPI2002-03958, TEC2006-03863 and TEC2007-61879.

\section{REFERENCES}

[1] Shun-Chung Wang, Yi-Hua Liu, Yih Chien Chen, Juing Huei Su and Wei-Sibge Liaw, "Web-based software programmable e-learning platform for power electronics courses," in $38^{\text {th }}$ Annual Frontiers in Education Conference (FIE 2008), pp. T2B-9-T2B-14, 22-25 Oct. 2008.

[2] M. P. Kazmierkowski, R. Bracha and M. Malinowski, "Web Based Teaching of Pulse Width Modulation Methods for Three-Phase TwoLevel Converters," in $12^{\text {th }}$ International Power Electronics and Motion Control Conference (EPE-PEMC 2006), pp. 2134-2139, Aug. 30-Sept. 12006.

[3] V. Leite, J. Barbosa, H. Teixeira and R. E. Araujo, "A generic converter for experimentation based power electronics learning," in 2005 European Conference on Power Electronics and Applications, pp. 1-10, 2005.

[4] P. Gamboa, J. Faria, E. Margato, J. Palma and L. Ferreira, "Modern technologies for experimental education in industrial electronics and electric drives," in 2005 European Conference on Power Electronics and Applications, pp. 1-8, 2005.

[5] R. S. Balog, Z. Sorchini, J. W. Kimball, P. L. Chapman and P. T. Krein, "Modern laboratory-based education for power electronics and electric machines," IEEE Trans. Power Systems, vol. 20, no. 2, pp. 538-547, May 2005

[6] H. Widlok and M. Widlok, "Computer-Aided Teaching of Power Electronics," in 12th International Power Electronics and Motion Control Conference (EPE-PEMC 2006), pp. 1733-1736, Aug. 30 2006-Sept. 1 2006.

[7] A. Nogueiras, A. Lago, L. Eguizabal, J. Marcos and C. MartinezPenalver, "Power Electronics Education: Lectures, Simulation and Laboratory Sinergetic Approach," in the International Conference on Computer as a Tool (EUROCON 2005), vol. 1, pp. 827-830, 21-24 Nov. 2005.

[8] V. G. Agelidis, "The future of power electronics/power engineering education: challenges and opportunities," in 2005 IEEE Workshop on Power Electronics Education, pp. 1-8, June 16-17, 2005.

[9] Interactive Power Electronics Seminar located in http://www.ipes.ethz.ch/ipes/e_index.html.

[10] P. Bauer, "Remote controlled practical education for power electronics," in 2007 European Conference on Power Electronics and Applications, pp. 1-10, 2007.

[11] Shun-Chung Wang and Yi-Hwa Liu, "Software-Reconfigurable eLearning Platform for Power Electronics Courses," IEEE Trans. Industrial Electron., vol. 55, no. 6, pp. 2416-2424, June 2008. 